\title{
Compressive Spectral Imaging with Diffractive Lenses
}

\author{
OĞUZhan FATIH KAR ${ }^{1}$ ANd Figen S. OKTEM ${ }^{1}{ }^{*}$ \\ ${ }^{1}$ Department of Electrical and Electronics Engineering, Middle East Technical University (METU), Ankara, 06800, Turkey \\ *Corresponding author: figeno@metu.edu.tr
}

Compressive spectral imaging enables to reconstruct the entire 3D spectral cube from a few multiplexed images. Here, we develop a novel compressive spectral imaging technique using diffractive lenses. Our technique uses a coded aperture to spatially modulate the optical field from the scene and a diffractive lens such as a photon-sieve for both dispersion and focusing. Measurement diversity is achieved by changing the focusing behavior of the diffractive lens. The 3D spectral cube is then reconstructed from highly compressed measurements taken with a monochrome detector. A fast sparse recovery method is developed to solve this large-scale inverse problem. The performance is illustrated at visible regime for various scenarios with different compression ratios through simulations. The results demonstrate that promising reconstruction performance can be achieved at high compression levels. This opens up new possibilities for high resolution spectral imaging with low-cost and simpler designs. (๑) 2019

Spectral imaging is a fundamental diagnostic technique in physical sciences with application in diverse fields such as physics, chemistry, biology, medicine, astronomy, and remote sensing. Conventional techniques rely on a scanning process to build up the 3D spectral cube from a series of 2D measurements [1]. One important disadvantage is that higher number of scans is needed with increased spatial and spectral resolutions [2]. This may lead to low light throughput, increased hardware complexity, and long acquisition times, resulting in temporal artifacts in dynamic scenes. Moreover, the temporal, spatial, and spectral resolutions are inherently limited as they are purely determined by the physical systems involved.

Compressive spectral imaging provides an effective way to overcome these limitations by passing on some of the burden to a computational system. It enables to reconstruct the entire spectral cube from a few multiplexed measurements via sparse recovery. This is made possible by compressive sensing (CS) which relies on two principles: sparsity of the spectral images in a transform domain and incoherence of the measurements. It is widely known that spectral images exhibit both spatial and spectral correlations, which allow sparse representations [2]. For the incoherence of the measurements, different computational spectral imaging techniques have been proposed, as reviewed in $[2,3]$. Examples include coded aperture snapshot spectral imaging (CASSI) and its variants [2, 4-6], and compressive hy-

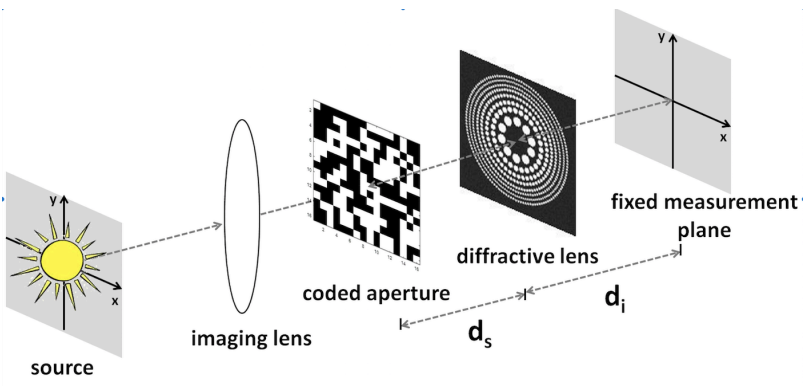

Fig. 1. Illustration of the CSID system.

perspectral imaging by separable spectral-spatial operators [7].

In this letter, we develop a novel compressive spectral imaging technique named compressive spectral imaging with diffractive lenses (CSID). CSID uses a coded aperture to spatially modulate the optical field from the scene and a diffractive lens such as a photon sieve $[8,9]$ for both dispersion and focusing. The coded field is first passed through the diffractive lens and then recorded with a monochromatic detector. Measurement diversity is achieved by changing the focusing behavior of the diffractive lens. A novel fast sparse recovery method is also developed to reconstruct the spectral cube from compressive measurements. The performance is illustrated numerically for various settings.

Different than the earlier works that use diffractive lenses for spectral imaging [10-12], here we utilize them for the first time in a compressive modality. Moreover, our system performs dispersion and focusing with a single element (a diffractive lens) unlike conventional imaging spectrometers and computational spectral imaging systems like CTIS [1] and CASSI [4] for which collimating and focusing optics are also required in addition to a disperser (grating/prism). Since diffractive lenses are also lightweight and low-cost to manufacture for a wide spectral range including x-rays and UV [8,17], our approach enables high resolution spectral imaging with simpler and low-cost designs.

Figure 1 illustrates the CSID system, which consists of (1) an imaging lens, (2) a coded mask, (3) a diffractive lens (such as a photon sieve), and (4) a monochrome detector [13]. First the image of the scene is formed on the plane of the coded mask, and then the coded field is passed through the diffractive lens. Since the diffractive lens has a wavelength-dependent focal length, each spectral component is exposed to a different amount of focus. As a result, each measurement is a superposition of differently blurred and coded spectral bands. To achieve measurement diversity, a total of $K$ such measurements can be recorded by changing the focusing behavior of the diffractive lens. Such few measurements can be obtained in different ways, such as 
with a programmable diffractive lens realized by a rapidly varying commercial spatial light modulator or digital micromirror device (DMD), or in a snapshot using multiple diffractive lenses and beam splitters.

The measurements obtained with the CSID system can be related to the intensity of each spectral component as follows:

$$
y_{k}(u, v)=\int\left(f_{\lambda}(u, v) * h_{\lambda, k}(u, v)\right) b(\lambda) d \lambda .
$$

Here $y_{k}(u, v)$ represents the $k$ th measurement, $f_{\lambda}(u, v)=$ $x_{\lambda}\left(-\frac{d_{s}}{d_{i}} u,-\frac{d_{s}}{d_{i}} v\right) c_{\lambda}\left(-\frac{d_{s}}{d_{i}} u,-\frac{d_{s}}{d_{i}} v\right)$ is the coded and scaled intensity of the spectral field $x_{\lambda}(u, v)$ with coded aperture $c_{\lambda}(u, v)$. Assuming an ideal imaging lens with unit magnification, this coded and scaled intensity is convolved with the incoherent point-spread function (PSF) of the $k$ th diffractive lens, $h_{\lambda, k}(u, v)$, which has a a closed-form expression given elsewhere [14]. Lastly, $b(\lambda)$ denotes the spectral response of the detector.

We discretize the spectral field into $S$ spectral bands, and $x_{s}(u, v)$ represents the intensity of the sth band with central wavelength $\lambda_{s}$. This spectral component is modulated with the coded mask pattern $c_{S}(u, v)$ at $\lambda_{S}$. The patterns $c_{S}(u, v)$ are the same for all wavelengths $(s=1, \ldots, S)$ if an uncolored (blockunblock) mask is used; however, these will be different if a colored coded mask [2] is used instead. The coded aperture $c_{s}(u, v)=\sum_{m, n} c_{s}[m, n] \operatorname{rect}\left(\frac{u}{\Delta_{c}}-m, \frac{v}{\Delta_{c}}-n\right)$ is a pixelated array with a pixel size of $\Delta_{c}$, and $c_{\mathcal{S}}[m, n]$ denotes the value of the coded aperture at pixel $(m, n)$.

After discretizing the field along the spectral dimension, discretization along the spatial dimensions is also needed to arrive at a discrete model. Replacing each spatially continuous function with its discretized version, we obtain the following model:

$$
y_{k}[m, n]=\sum_{s=1}^{S}\left(b_{s} x_{s}[m, n] c_{s}[m, n]\right) * h_{\lambda_{s}, k}[m, n] .
$$

Here, $y_{k}[m, n]$ denotes the $k$ th measurement obtained over $N_{x} \times$ $N_{y}$ detector pixels, and corresponds to the samples of $y_{k}(u, v)$, i.e. $y_{k}[m, n]=y_{k}(m \Delta, n \Delta)$. The sampling interval $\Delta$ is equal to the pixel size of the detector. The coded aperture pixel size can be chosen as an integer multiple of $\Delta$ to avoid the need for subpixel positioning accuracy. Here, we choose $\Delta_{c}=\Delta$ for simplicity. Moreover, $x_{s}[m, n]$ and $h_{\lambda_{s}, k}[m, n]$ are the uniformly sampled versions of their continuous counterparts with the same sampling interval $\Delta$. Lastly, $b_{s}$ represents the coefficient resulting from the response of the detector at the central wavelength $\lambda_{s}$.

This discrete model can be expressed in the following form:

$$
\mathbf{y}=\mathbf{H C x}+\mathbf{n},
$$

where $\mathbf{y}=\left[\mathbf{y}_{1}^{T}, \ldots, \mathbf{y}_{K}^{T}\right]^{T} \in \mathbb{R}^{K N}$ is vertically concatenated measurement vector with $N \triangleq N_{x} N_{y}$ and $\mathbf{y}_{k} \in \mathbb{R}^{N}$ denoting the $k$ th measurement vector. Similarly, $\mathbf{x}=\left[\mathbf{x}_{1}^{T}, \ldots, \mathbf{x}_{S}^{T}\right]^{T} \in \mathbb{R}^{S N}$ is the concatenated image vector with $\mathbf{x}_{S} \in \mathbb{R}^{N}$ denoting the spectral image vector at wavelength $\lambda_{s}$. The $K N \times S N$ matrix $\mathbf{H}$ consists of $N \times N$ convolution matrices representing the convolutions with PSFs $h_{\lambda_{s}, k}[m, n]$. The diagonal matrix $\mathbf{C} \in \mathbb{R}^{S N \times S N}$ performs the overall coding operation, and has values 0 or 1 along its diagonal. Finally, the vector $\mathbf{n}=\left[\mathbf{n}_{1}^{T}, \ldots, \mathbf{n}_{K}^{T}\right]^{T}$ denotes the noise, which is often white Gaussian. In our setting, the number of measurements $(K)$ is smaller than the number of spectral bands $(S)$, which results in an under-determined system.

In the inverse problem, the goal is to reconstruct the unknown spectral images, $\mathbf{x}$, from their compressive superimposed measurements, $\mathbf{y}$, which contain their coded and blurred versions.
This problem is inherently ill-posed. There are a variety of approaches to solve such ill-posed linear inverse problems. Here, to exploit the sparsity of the spectral images after some transformation $\Phi$, we formulate the inverse problem as the following constrained optimization problem:

$$
\min _{\mathbf{x}}\|\Phi \mathbf{x}\|_{1} \text { subject to }\|\mathbf{y}-\mathbf{H C} \mathbf{x}\|_{2} \leq \epsilon,
$$

where $\epsilon \geq 0$ is a parameter that depends on noise variance. Here $\ell_{1}$-norm enforces the sparsity of the spectral cube after transformation with $\Phi$, as motivated by the CS theory.

To solve the resulting optimization problem, we convert our constrained problem to an unconstrained problem by adding the constraint to the objective function as a penalty function:

$$
\min _{\mathbf{x}}\|\Phi \mathbf{x}\|_{1}+\iota_{\left(\|\mathbf{y}-\mathbf{H C} \mathbf{x}\|_{2} \leq \epsilon\right)}(\mathbf{x}),
$$

where the indicator function $\iota_{\left(\|\mathbf{y}-\mathbf{H C} \mathbf{x}\|_{2} \leq \epsilon\right)}(\mathbf{x})$ takes value 0 if the constraint is satisfied, and $+\infty$ otherwise. We solve this problem by developing a fast reconstruction algorithm that is based on alternating direction method of multipliers (ADMM) [15]. After variable-splitting, we arrive at the following problem:

$$
\begin{array}{cc}
\underset{\mathbf{x}, \mathbf{z}^{(1)}, \mathbf{z}^{(2)}}{\operatorname{minimize}} & \left\|\Phi \mathbf{z}^{(1)}\right\|_{1}+\iota_{\left(\left\|\mathbf{y}-\mathbf{z}^{(2)}\right\|_{2} \leq \epsilon\right)}\left(\mathbf{z}^{(2)}\right) \\
\text { subject to } & \mathbf{z}^{(1)}=\mathbf{x}, \quad \mathbf{z}^{(2)}=\mathbf{H C} \mathbf{x}
\end{array}
$$

where $\mathbf{z}^{(1)}, \mathbf{z}^{(2)}$ are the auxiliary variables in the ADMM framework. After expressing the problem in Eq. (6) in augmented Lagrangian form [15], minimization over $\mathbf{x}, \mathbf{z}^{(1)}$, and $\mathbf{z}^{(2)}$ is needed. Here, we minimize over each in an alternating fashion.

For minimization over $\mathbf{x}$, we face a least-squares problem which has the following normal equation:

$$
\left(\mathbf{I}+\mathbf{C} \mathbf{H}^{H} \mathbf{H C}\right) \mathbf{x}_{k+1}=\left(\mathbf{z}^{(1)}+\mathbf{d}^{(1)}+\mathbf{C} \mathbf{H}^{H}\left(\mathbf{z}^{(2)}+\mathbf{d}^{(2)}\right)\right)
$$

with $\mathbf{d}$ denoting the dual variable in the ADMM framework. A direct matrix inversion approach for solving the linear system in Eq. (7) is not feasible for large-scale spectral cubes. Here, we solve this iteratively using the conjugate-gradient method. For this iterative process, forming any of the matrices is not required, which provides huge savings for the memory and computation time. Specifically, multiplications with matrices $\mathbf{H}$ and $\mathbf{H}^{H}$ correspond to summation of some convolutions. That is, for multiplication with $\mathbf{H}$ matrix, we simply take 2D Fourier transforms of underlying PSFs $h_{\lambda_{1}, k}[m, n], \ldots, h_{\lambda_{S}, k}[m, n]$ and the spectral images $x_{1}[m, n], \ldots, x_{S}[m, n]$, multiply them element-wise, and then sum. For multiplication with $\mathbf{H}^{H}$ matrix, a similar operation is performed using the PSFs $h_{\lambda_{s}, 1}[m, n], \ldots, h_{\lambda_{s}, K}[m, n]$. Moreover, the multiplication with $\mathrm{C}$ corresponds to simple element-wise multiplications with coded apertures $c_{S}[m, n]$.

Minimization over $\mathbf{z}^{(1)}$ requires the following operation:

$$
\mathbf{z}_{k+1}^{(1)}=\Phi^{-1}\left(\operatorname{soft}\left(\Phi\left(\mathbf{x}_{k+1}-\mathbf{d}_{k}^{(1)}\right), \frac{1}{\mu}\right)\right),
$$

Here, $\operatorname{soft}(\mathbf{w}, \tau)$ denotes the soft-thresholding operation and is component-wise computed as $\mathbf{w}_{i} \rightarrow \operatorname{sign}\left(\mathbf{w}_{i}\right) \max \left(\left|\mathbf{w}_{i}\right|-\tau, 0\right)$ for all $i$, with $\operatorname{sign}\left(\mathbf{w}_{i}\right)$ taking value 1 if $w_{i}>0$ and -1 otherwise [15]. That is, the solution in Eq. (8) can be obtained through transformation with $\Phi$, followed by soft-thresholding with parameter $1 / \mu$, and inverse transformation operation $\Phi^{-1}$. 
For minimization over $\mathbf{z}^{(2)}$, a projection of $\mathbf{s} \triangleq\left(\mathbf{H C} \mathbf{x}_{k+1}-\right.$ $\mathbf{d}_{k}^{(2)}$ ) onto $\epsilon$-radius hypersphere centered at $\mathbf{y}$ is required [15]. This projection has the following form:

$$
\mathbf{z}_{k+1}^{(2)}= \begin{cases}\mathbf{y}+\epsilon \frac{\mathbf{s}-\mathbf{y}}{\|\mathbf{s}-\mathbf{y}\|_{2}}, & \text { if }\|\mathbf{s}-\mathbf{y}\|_{2}>\epsilon \\ \mathbf{s}, & \text { if }\|\mathbf{s}-\mathbf{y}\|_{2} \leq \epsilon .\end{cases}
$$

As a result, we have three update steps resulting from the ADMM formulation, i.e. $\mathbf{x}$-update, $\mathbf{z}^{(1)}$-update, and $\mathbf{z}^{(2)}$-update. The overall algorithm is summarized in Table 1.

Table 1. Reconstruction algorithm for CSID

Input: Compressive measurements $\mathbf{y}$ as in Eq. (3).

Initialization: Iteration count $k=0$, choose $\mu>0, \epsilon$, $\mathbf{z}_{0}^{(1)}, \mathbf{z}_{0}^{(2)}, \mathbf{d}_{0}^{(1)}, \mathbf{d}_{0}^{(2)}$.

Main Iteration: Repeat until stopping criterion satisfied.

1. Calculate spectral images $\mathbf{x}_{k+1}$ by solving Eq. (7) using conjugate-gradient algorithm.

2. Calculate $\mathbf{z}_{k+1}^{(1)}$ using soft-thresholding in Eq. (8).

3. Calculate $\mathbf{z}_{k+1}^{(2)}$ using projection in Eq. (9).

4. Update $\mathbf{d}_{k+1}^{(1)}$ as $\mathbf{d}_{k+1}^{(1)}=\mathbf{d}_{k}^{(1)}-\left(\mathbf{x}_{k+1}-\mathbf{z}_{k+1}^{(1)}\right)$.

5. Update $\mathbf{d}_{k+1}^{(2)}$ as $\mathbf{d}_{k+1}^{(2)}=\mathbf{d}_{k}^{(2)}-\left(\mathbf{H C} \mathbf{x}_{k+1}-\mathbf{z}_{k+1}^{(2)}\right)$.

Output: Spectral images $\mathbf{x}$.

We now present numerical simulations to illustrate the performance and compare with CASSI. We consider a spectral dataset of size $820 \times 820 \times 31$ ( 31 wavelengths between $410-710$ $\mathrm{nm}$ with $10 \mathrm{~nm}$ interval), taken from an online hyperspectral database [16] and referred as Objects data in a CASSI work [5]. For this dataset, 25 outlier voxel values are dropped to 0.3 , and the spectral cube is scaled to $[0,1]$. As the diffractive lens, photon sieves are used with a smallest hole diameter of $\delta=8 \mu \mathrm{m}$, providing Abbe's spatial resolution of $8 \mu \mathrm{m}$. Pixel size of the detector, $\Delta$, is chosen as $4 \mu \mathrm{m}$ to match this spatial resolution.

Measurements are taken by changing the focusing behavior of the photon sieve. For each measurement, the detector to diffractive lens distance is fixed as $f_{0}=2.56 \mathrm{~cm}$, and the design is changed to focus a different wavelength at this distance. For this purpose, the outer diameter of the sieve is changed as $D_{k}=\tilde{\lambda}_{k} f_{0} / \delta$ [17], where $\tilde{\lambda}_{k}$ is the wavelength focused at the $k$ th measurement. For example, if $\tilde{\lambda}_{k}=560 \mathrm{~nm}$, the diameter $D_{k}=1.8 \mathrm{~mm}$. Moreover, the expected spectral resolution is $4 \delta^{2} / f_{0}=10 \mathrm{~nm}$, as given by the spectral bandwidth of the diffractive lens [17][Chap. 9]. Note that this expected spectral resolution matches to the spectral sampling interval, i.e. $10 \mathrm{~nm}$.

The compressive measurements are simulated using the model in Eq. (3) with additive Gaussian noise. In each measurement, the system applies the same masking operation to each spectral band using a traditional block-unblock mask, whose entries are drawn from a Bernoulli distribution as shown in Fig. 2. After the coded field passes through the photon sieve, we capture measurements at the same plane by changing the outer diameter of the sieve. A sample compressive measurement is shown in Fig. 2 together with the true spectral cube superimposed along the spectral dimension. In this measurement, $430 \mathrm{~nm}$ is focused by the sieve onto the detector plane, while all other spectral components are defocused. To illustrate this, we also provide the acting PSFs for three spectral components, which show the different amount of blur. As seen, the measurements involve not only the superposition of all spectral bands but also significant amount of blur and degradation.

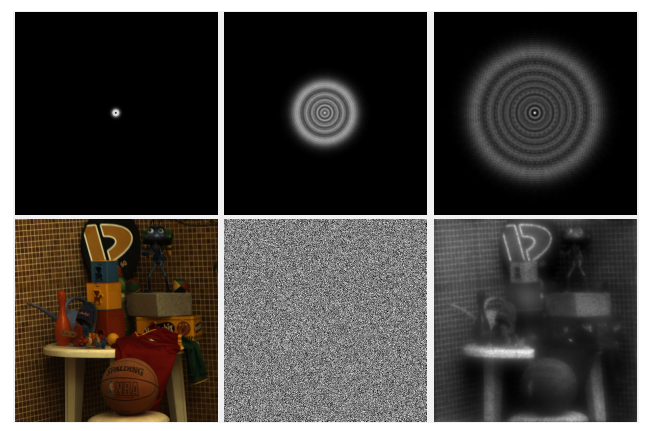

Fig. 2. Demonstration of compressive measurements for $K=3$ case. In this measurement, $430 \mathrm{~nm}$ is focused onto the detector plane, while all other spectral components are defocused. Top row: PSFs of the photon-sieve for spectral components at 410 $\mathrm{nm}, 560 \mathrm{~nm}$, and $710 \mathrm{~nm}$. Bottom row: Superimposed true image, sample mask pattern, sample compressive measurement.

Table 2. Comparison of reconstruction PSNRs (dB) / SSIMs / SAMs for different compressive scenarios and SNRs.

\begin{tabular}{|c|c|c|c|}
\hline SNR (dB) & $K=2$ & $K=3$ & $K=4$ \\
\hline 22 & $28.62 / 0.73 / 19.3^{\circ}$ & $32.13 / 0.84 / 12.4^{\circ}$ & $32.60 / 0.85 / 11.8^{\circ}$ \\
\hline 28 & $28.93 / 0.73 / 18.9^{\circ}$ & $32.73 / 0.85 / 11.8^{\circ}$ & $33.42 / 0.87 / 10.9^{\circ}$ \\
\hline 34 & $29.23 / 0.73 / 18.7^{\circ}$ & $33.16 / 0.86 / 11.4^{\circ}$ & $34.19 / 0.88 / 10.2^{\circ}$ \\
\hline
\end{tabular}

We consider different compressive scenarios with $K=2,3$ and 4 measurements. For each case, equidistant wavelengths from the spectral range $410-710 \mathrm{~nm}$ are chosen to be focused onto the detector plane. More specifically, the chosen wavelengths are $\{500,610\} \mathrm{nm}$ for $K=2,\{430,560,680\} \mathrm{nm}$ for $K=3$, and $\{420,510,600,690\} \mathrm{nm}$ for $K=4$. These cases with $K=2,3,4$ correspond to compression levels (CLs), $100 \times(1-K / S)$, of $93.5 \%, 90.3 \%$ and $87.1 \%$, respectively. These are equivalent to reconstructing the spectral cube from $6.5 \%, 9.7 \%$ and $12.9 \%$ data.

To analyze medium to low noise cases, input SNRs of 22, 28 , and $34 \mathrm{~dB}$ are considered, by adding Gaussian noise with standard deviation equal to $1 \%, 0.50 \%$, and $0.25 \%$ of the maximum value in the noiseless measurements. Reconstructions are obtained from these compressive noisy measurements using the algorithm in Table 1. Similar to previous compressive spectral imaging approaches [2], we enforce sparsity in a Kronecker basis $\Phi=\Phi_{1} \otimes \Phi_{2}$ where $\Phi_{1}$ is the basis for 2D Symmlet- 8 wavelet and $\Phi_{2}$ is the 1D discrete cosine (DCT) basis. This transformation is computed by first taking the 2D Symmlet- 8 transform of each spectral image and then 1D DCT along the spectral dimension.

The average reconstruction performance for all cases is given in Table 2 in terms of PSNR, SSIM, and spectral angular mapper (SAM). As seen, PSNR is above $28.5 \mathrm{~dB}$, SSIM is above 0.73 , and SAM is less than $19.3^{\circ}$ for all cases, which demonstrates faithful reconstruction even at high compression levels. Moreover, for $K=3$ case (i.e. reconstruction from $9.7 \%$ data), PSNR is greater than $32.13 \mathrm{~dB}$ and SSIM is greater than 0.84 for all three SNRs. These values are better than the multi-frame CASSI results for the same dataset and compression level given in [5] (PSNR=27.04 $\mathrm{dB}, \mathrm{SSIM}=0.82$ ). In addition, the performance degrades gracefully with decreased SNR and increased compression.

To visually evaluate the results, we provide in Fig. 3 the reconstructed spectral images at different compression levels for $\mathrm{SNR}=28 \mathrm{~dB}$, together with the true images. As seen, the image details and edges, as well as the spectral variations, are well preserved in the reconstructions. In the left of each row, superimposed spectral cube is also shown, which is similar to the true 


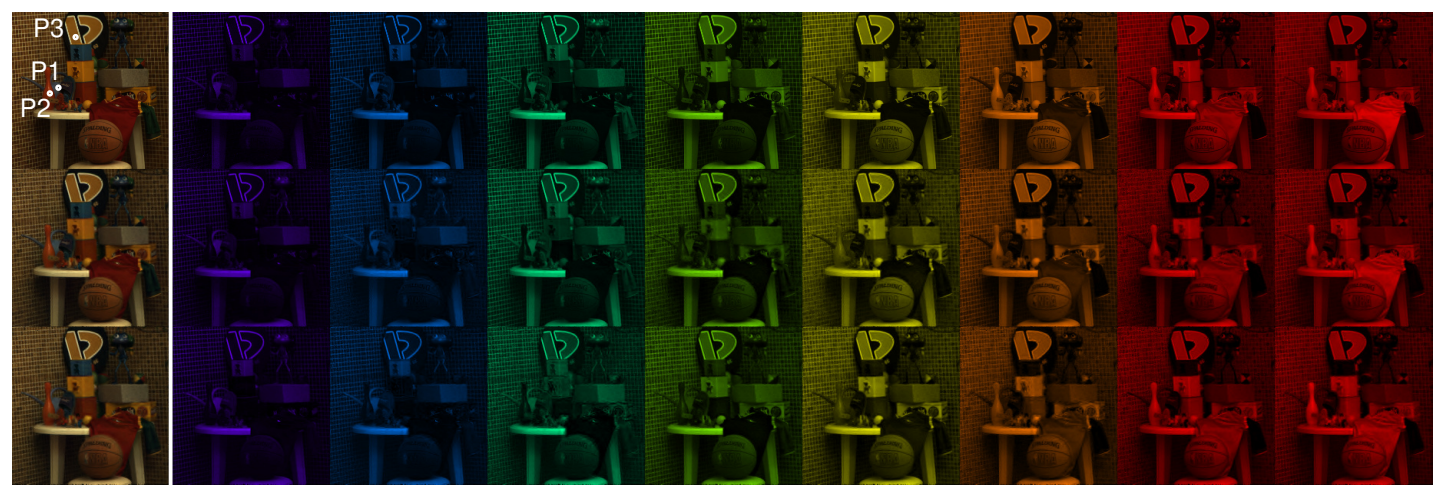

Fig. 3. Sample reconstructed images from compressive measurements when $\mathrm{SNR}=28 \mathrm{~dB}$. In the left of each row, superimposed spectral cube along the spectral dimension is shown; other columns contain spectral images at wavelengths 420, 460, 500, 540, 580, 620,660 , and $700 \mathrm{~nm}$. Top to bottom: true images, reconstructions with $K=4$ and $K=3$ measurements.
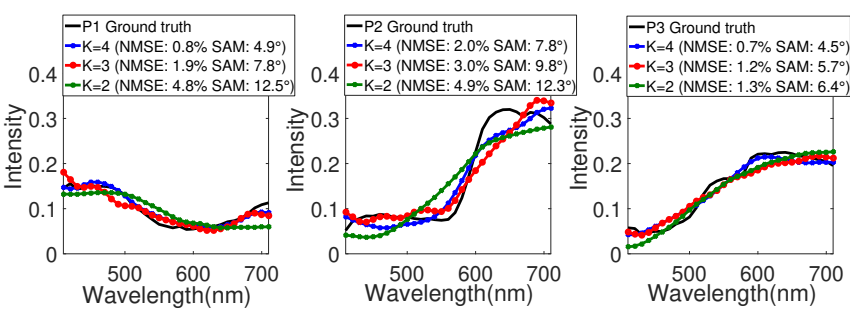

Fig. 4. Spectrum at the points $\mathrm{P} 1, \mathrm{P} 2, \mathrm{P} 3$ for $\mathrm{SNR}=28 \mathrm{~dB}$.

one. Hence, the results demonstrate successful reconstruction of the spectral cube at compression levels as high as $\sim 90 \%$.

To also demonstrate the successful recovery along the spectral dimension, we select three representative points with different spectral characteristics, as shown as P1, P2, and P3 in Fig. 3. The reconstructed spectra at these points are plotted in Fig. 4, together with the ground truth. As seen, the spectrum is recovered successfully at all compression ratios for each point. To numerically evaluate the spectrum recovery, SAM and percentage mean squared error (NMSE) values are also given in the legends.

In these results, the pixel size of the detector and the reconstruction grid are chosen to match the expected spatial resolution of the diffractive lens. Because the developed modality is based on computational imaging and compression is performed along the spectral direction, effective spectral resolution not only depends on the diffractive lens design, but also on the scene content (i.e. the spectral correlation). Although the imaging performance appears to be robust to higher compression levels and noise, clearly increasing the number of measurements improves the reconstructions. However, this comes with the cost of increased acquisition time (i.e. undesirable for dynamic scenes).

In summary, we have presented a novel compressive spectral imaging modality with a simple optical configuration involving a coded aperture and a diffractive lens. Together with the developed reconstruction algorithm, promising imaging performance is achieved even at high compression levels. Since the system performs compression along the spectral dimension, successful reconstructions can be obtained for spectrally-correlated scenes. Although the presented results are for the visible range, the imaging concept is equally applicable to other regimes as well. Moreover, the part of the imaging system after the coded aperture is shift-invariant unlike earlier systems. This enables easier design, faster reconstruction, and simpler calibration. In particular, for calibration, measuring the PSFs is sufficient, instead of the system response for each voxel. The performance can be further improved with the use of colored coded apertures. Future work will focus on the experimental demonstration.
Different than the earlier compressive spectral imagers that rely on prisms/gratings to disperse the optical field and require additional collimating/re-imaging optics, we use a single diffractive lens to achieve both dispersion and focusing. Moreover, unlike conventional collimating/imaging optics, diffractive lenses are lightweight and low-cost to manufacture for a wide spectral range including $x$-rays and UV. Hence this work opens up new possibilities for high resolution spectral imaging with low-cost and simpler designs in a wide range of applications.

Funding: Scientific and Technological Research Council of Turkey (TUBITAK), 3501 Research Program, 117E160.

\section{REFERENCES}

1. T. Okamoto and I. Yamaguchi, Opt. Lett. 16, 1277 (1991).

2. X. Cao, T. Yue, X. Lin, S. Lin, X. Yuan, Q. Dai, L. Carin, and D. J. Brady, IEEE Signal Process. Mag. 33, 95-108 (2016).

3. F. S. Oktem, L. Gao, and F. Kamalabadi, "Computational spectral and ultrafast imaging via convex optimization," in Handbook of Convex Optimization Methods in Imaging Science, (Springer, 2018), pp. 105127.

4. A. Wagadarikar, R. John, R. Willett, and D. Brady, Appl. Opt. 47, B44 (2008).

5. A. Rajwade, D. Kittle, T.-H. Tsai, D. Brady, and L. Carin, SIAM J. on Imaging Sci. 6, 782-812 (2013).

6. E. Salazar, A. Parada-Mayorga, and G. R. Arce, IEEE Transactions on Comput. Imaging 5, 165-179 (2019).

7. Y. August, C. Vachman, Y. Rivenson, and A. Stern, Appl. Opt. 52, D46 (2013).

8. F. S. Oktem, F. Kamalabadi, and J. M. Davila, "High-resolution computational spectral imaging with photon sieves," in IEEE ICIP, (IEEE, 2014), pp. 5122-5126.

9. G. Andersen, Opt. Lett. 30, $2976(2005)$.

10. P. Wang and R. Menon, JOSA A 35, 189 (2018).

11. F. D. Hallada, A. L. Franz, and M. R. Hawks, Opt. Eng. 56, 081811 (2017).

12. M. Nimmer, G. Steidl, R. Riesenberg, and A. Wuttig, Opt. Express 26, 28335 (2018).

13. O. F. Kar, U. Kamaci, F. C. Akyon, and F. S. Oktem, "Compressive photon-sieve spectral imaging," in Computational Optical Sensing and Imaging, (Optical Society of America, 2018), pp. CTu5D-8.

14. F. S. Oktem, F. Kamalabadi, and J. M. Davila, Opt. Express 26, 32259 (2018).

15. M. V. Afonso, J. M. Bioucas-Dias, and M. A. T. Figueiredo, IEEE Trans. Image Process. 20, 681 (2011).

16. S. M. Nascimento, F. P. Ferreira, and D. H. Foster, JOSA A 19, 1484 (2002).

17. D. Attwood, Soft $x$-rays and extreme ultraviolet radiation: principles and applications (Cambridge University Press, 2000). 